\title{
Evaluation of Ethiopian Commercial Durum and Bread Wheat Varieties, Candidate and Differential Lines against Yellow Rust
}

\section{4-2016}

\author{
Kasa D*, Negash T and Yirga F \\ Ethiopian Institute of Agricultural Research (EIAR), Kulumssa Agricultural Research \\ Center, P.0. Box. 489, Asella, Ethiopia
}

*Corresponding author: Daniel Kasa, Ethiopian Institute of Agricultural Research (EIAR),

Kulumssa Agricultural Research Center, P.O. Box. 489, Asella, Ethiopia, Tel: +251917850880; Email: danikasa2008@gmail.com

\section{Research Article}

Volume 3 Issue 3

Received Date: May 09, 2018

Published Date: May 24, 2018

\section{Abstract}

Yellow rust, caused by Puccinia striiformis f. sp. tritici is one of the most damaging diseases of wheat in worldwide. In Ethiopia, grain yield loss in wheat cultivars ranges from 30 to 69\%. The highland of Ethiopia is considered as a hot spot for the development of yellow rust diversity. This study was carried out to monitor commercial cultivars and advanced lines of bread and durum wheat for their resistance to the prevailing race population and to identify effective resistance Yr genes. One hundred four (104) commercial and candidate varieties of durum and bread wheat were tested $96.2 \%$ of them were infected with yellow rust at different severity level (trace to 90S). The peak average severity of stripe rust on commercial and candidate varieties of bread and durum wheat reached with the range of 53.3 to $63.3 \%$ and 0 to $37 \%$ respectively. The result of yellow rust differential line indicated that most of the Yr (yellow rust) gene are not effective across all locations like Yr1, Yr5, Yr6, Yr7, Yr8, Yr9, Yr 10, Yr17, Yr18, Yr26, Yr27 and YrA under field condition. Whereas Yr15 and Lassik (-Yr5) were effective genes to all the prevailing isolate for the last three cropping season.

Keywords: Commercial Wheat Cultivars; Differential Lines; Effective Genes; Yellow Rust

\section{Introduction}

Yellow or stripe rust (Puccinia striiformis f.sp. tritici) is a major wheat disease in the highlands of Ethiopia. Arsi and Bale also the major wheat production zones of the country and the known hotspots for the epidemics of yellow rust of wheat [1]. Even though there is seasonal variability in the occurrence of yellow rust in Bale highlands, the main and long rainy season is ideal for yellow rust development [2]. 58 percent of yield loss was recorded at Bekoji in 1988 due to yellow rust epidemics on Dashen. Grain yield losses of 30 to $96 \%$ have been recorded on susceptible bread wheat varieties in Bale [3]. Grain yield loss of $71 \%$ has been recorded on susceptible bread wheat variety Wabe in Bale [4]. Yellow rust also reduced the germination ability and kernel weight of Dashen by 72 and $56 \%$ respectively [5]. Wheat researchers in Ethiopia have been continuously breeding for disease resistance, wide adaptability and high yield, which resulted in the release of many cultivars to farmers. However, most of these cultivars were abandoned from production due to their susceptibility mainly to yellow 


\section{Open Access Journal of Agricultural Research}

rust disease [6]. The main reasons for periodic outbreaks of yellow rust disease in Ethiopia are the scarce information on the genetic variation of host-pathogen interactions and unreliability of current sources of resistance to the prevailing race population [5]. Breeding for resistance to wheat rusts has remained environmentally safe and economically feasible method of controlling the diseases. The knowledge of pathogenic specialization of rusts at host cultivar level help breeders and pathologists in providing host materials having useful gene (s) for resistance to the prevailing race population $[6,7]$. Therefore, bread and durum wheat cultivars, promising lines yellow rust differentials will be assembled to form the Ethiopian Wheat Rust Trap Nursery (EWRTN). The Objective this research paper was to monitor commercial cultivars and promising advanced lines of bread and durum wheat for their resistance to the prevailing race population and to identify effective resistance genes against the prevailing race population of the yellow rusts.

\section{Materials and Methods}

The trap nursery experiment was conducted in Meraro and Arsi robe district from 2014-2016. The experiment was composed of 104 commercial and candidate varieties of bread and durum wheat and 17 yellow rust differential hosts. Each entry was planted in non-replicated in two rows of $1 \mathrm{~m}$ spaced at $20 \mathrm{~cm}$. A spreader row consisting of susceptible checks of Morocco, PBW343 and Digalu were mixed in equal proportion to catch virulence for the $\mathrm{Yr}$ gene of stripe rust. Fertilizer was applied at the recommended rate for each location where the trap nurseries were planted. Scoring was done three times in each year during the growing season of 2014-2016 and only the maximum score per location was included in the report. The Modified Cobb's scale was used for scoring the severity of yellow rust of wheat and the response was recorded according to $[7,8]$.

\section{Results and Discussion}

\section{Reaction of Commercial and Candidate Varieties of Bread and Durum Wheat to Yellow Rust}

Of 83 bread and durum wheat varieties and lines evaluated $98.79 \%$ were infected with yellow rust with different average severity percentage range and reactions (0-63.3) and (trace - 90S) respectively at Arsi-robe and Meraro districts. Greater than 30 bread wheat cultivars and candidate lines that displayed resistant to moderately resistant reactions to the prevalent yellow rust races at both Arsi Robe and Meraro (Figure 1). The two most popular bread wheat varieties (Kubsa, and Digalu) in Ethiopia showed susceptible reaction comparing to the universally susceptible Morocco and PBW343. The bread wheat varieties and candidate line like Laketch, Mitike, Kubsa, Megal, Katar, Shina, Sulla, Kulkulu, ETBW6093, ETBW6647 and ETBW7255 exhibited susceptible reaction and the average severity level shows as $>30 \%$ at Arsi robe and Meraro within three cropping season. Among the infected genotypes, eleven (K6290 Bulk, K6295-4A, Pavon 76, Galema, Madda Walabu, Simba, Sofumar, Dodota, ETBW6861* (Lemu), Millennium, Hoggana, Kingbird and Hidassie exhibited average yellow rust severity range between $20-30 \%$ with a reaction of moderately resistant to susceptible. In addition, the varieties Enkoy, ET13A2, Meraro, Danda'a, Huluka, Gambo, Malefie, HAR719 and ETBW5800 shows range of $10-20 \%$ yellow rust severity in both locations. Among all commercial and candidate bread wheat varieties or lines that shows the lowest diseases severity range $0-10 \%$ was HAR727, HAR934, Bonny, Sanate, ETBW7698 and ETBW6130 (Wane) that displayed resistant to given yellow rust isolates with the reaction of moderately susceptible (MS) to moderately resistant (MR) in both location within three consecutive season (Table 2).

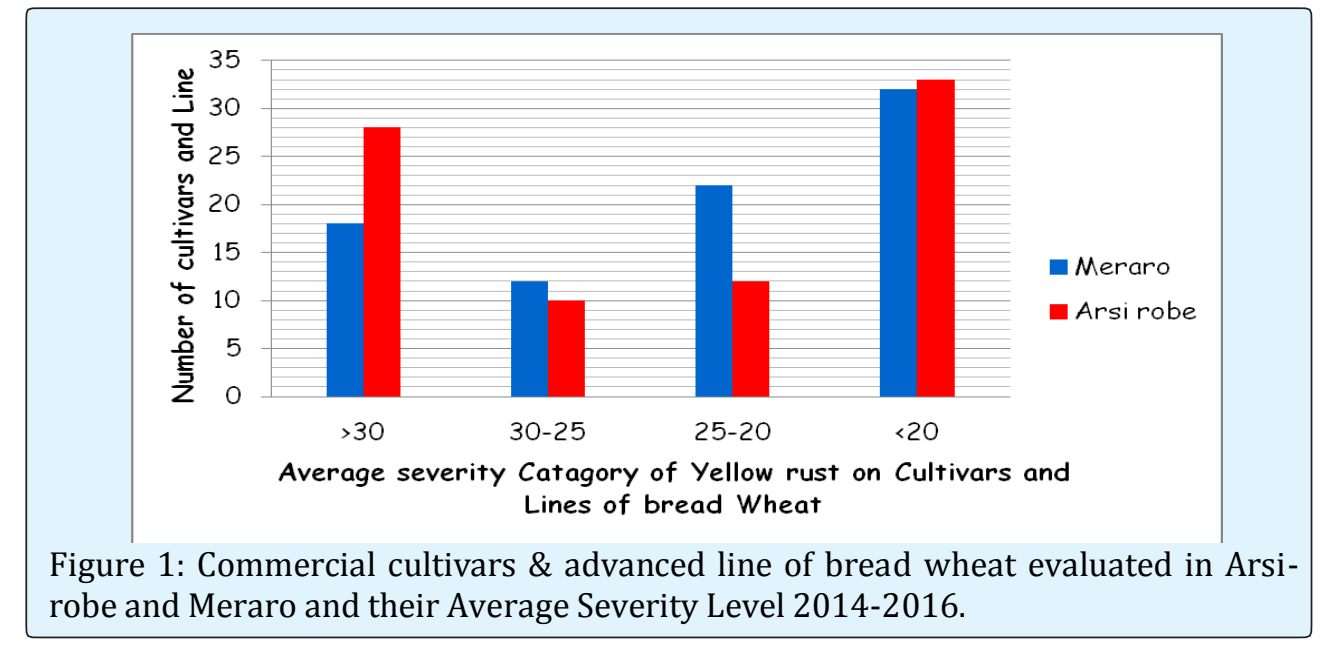

Kasa D, et al. Evaluation of Ethiopian Commercial Durum and Bread Wheat Varieties, Candidate and Differential Lines against Yellow Rust 2014-2016. J Agri Res 2018, 3(3): 000164. 


\section{Open Access Journal of Agricultural Research}

\begin{tabular}{|c|c|c|c|c|c|c|c|c|c|c|c|c|c|c|c|}
\hline \multirow{4}{*}{ Variety/line } & \multirow{4}{*}{$\mathrm{T}$} & \multirow{2}{*}{\multicolumn{6}{|c|}{$\begin{array}{l}\text { Severity and Reaction of Commercial } \\
\text { Variety and Lines for YR }\end{array}$}} & \multirow{4}{*}{ ASV\% } & \multicolumn{6}{|c|}{$\begin{array}{c}\text { Severity and Reaction of Commercial } \\
\text { Variety and Lines for YR }\end{array}$} & \multirow{4}{*}{ ASV\% } \\
\hline & & ARSI ROBE & & & & & & & \multicolumn{6}{|c|}{ MERARO } & \\
\hline & & \multicolumn{2}{|c|}{2014} & \multicolumn{2}{|c|}{2015} & \multicolumn{2}{|c|}{2016} & & \multicolumn{2}{|c|}{2014} & \multicolumn{2}{|c|}{2015} & \multicolumn{2}{|c|}{2016} & \\
\hline & & $\mathbf{S}$ & $\mathbf{R}$ & $\mathbf{S}$ & $\mathbf{R}$ & $\mathbf{S}$ & $\mathbf{R}$ & & $\mathbf{S}$ & $\mathbf{R}$ & $\mathbf{S}$ & $\mathbf{R}$ & $\mathbf{S}$ & $\mathbf{R}$ & \\
\hline Laketch & B & 50 & $\mathrm{~S}$ & 30 & $\mathrm{~s}$ & 80 & $\mathrm{~s}$ & 53.3 & 50 & $\mathrm{~s}$ & 60 & $\mathrm{~s}$ & 80 & $\mathrm{~s}$ & 63.3 \\
\hline Kenya Nyangumi & $\mathrm{B}$ & 20 & $\mathrm{~S}$ & 5 & sms & 30 & $\mathrm{~s}$ & 18.3 & 1 & tms & 60 & $\mathrm{~s}$ & 60 & $\mathrm{~s}$ & 40.3 \\
\hline Kenya Leopard & $\mathrm{B}$ & 1 & tr-ms & 5 & $\mathrm{~ms}$ & 1 & $\mathrm{mr}$ & 2.3 & 0 & 0 & 10 & sms & 30 & $\mathrm{~s}$ & 13.3 \\
\hline Africa Mayo & $\mathrm{B}$ & 10 & $\mathrm{~s}$ & 1 & tms & 10 & $\mathrm{mr}$ & 7 & 1 & tms & 20 & $\mathrm{~s}$ & 20 & $\mathrm{~s}$ & 13.7 \\
\hline Trophy & $\mathrm{B}$ & 10 & s-ms & 5 & $\mathrm{mr}$ & 20 & mrms & 11.7 & 1 & tms & 40 & $S$ & 50 & $\mathrm{~s}$ & 30.3 \\
\hline Bounty & $\mathrm{B}$ & 5 & $\mathrm{~s}$ & 5 & $\mathrm{~ms}$ & 30 & $\mathrm{~S}$ & 13.3 & 1 & tmrms & 10 & $\mathrm{~ms}$ & 50 & $s$ & 20.3 \\
\hline Bonny & $\mathrm{B}$ & 1 & tr-ms & 1 & tmr & 5 & $\mathrm{mr}$ & 2.3 & 1 & $\mathrm{tmr}$ & 1 & tms & 10 & $\mathrm{~ms}$ & 4 \\
\hline Frontach & B & 10 & s-ms & 5 & $\mathrm{msmr}$ & 50 & - & 21.7 & 5 & $\mathrm{msmr}$ & 20 & $\mathrm{~s}$ & 50 & $\mathrm{~s}$ & 25 \\
\hline Kenya Kudu & $\mathrm{B}$ & 5 & & 1 & & 30 & $\mathrm{~S}$ & 12 & 1 & tmrms & 20 & $\mathrm{mr}$ & 40 & $\mathrm{~s}$ & 20.3 \\
\hline Enkoy & $\mathrm{B}$ & 10 & $\mathrm{~ms}$ & 5 & S & 1 & $\mathrm{mr}$ & 5.3 & 5 & $\mathrm{~ms}$ & 10 & $\mathrm{~ms}$ & 20 & $\mathrm{~ms}$ & 11.67 \\
\hline K6290 Bulk & $\mathrm{B}$ & 20 & $\mathrm{~s}$ & 15 & & 50 & 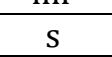 & 28.3 & 10 & is & 20 & $\mathrm{~s}$ & 60 & $\mathrm{~s}$ & 30 \\
\hline K6295-4A & B & 15 & $S$ & 10 & SIIIS & 60 & $\mathrm{~s}$ & 28.3 & 5 & $\mathrm{msmr}$ & 20 & $\mathrm{~ms}$ & 60 & $s$ & 28.3 \\
\hline ET13A2 & $\mathrm{B}$ & 5 & s-ms & 5 & $\mathrm{mr}$ & 50 & $\mathrm{~s}$ & 20 & 1 & $\mathrm{tmr}$ & 20 & $\mathrm{~s}$ & 30 & $\mathrm{~s}$ & 17 \\
\hline Pavon 76 & $\mathrm{~B}$ & 20 & $\mathrm{~S}$ & 30 & $\mathrm{~ms}$ & 40 & $\mathrm{~s}$ & 30 & 1 & tms & 20 & $\mathrm{~s}$ & 50 & $\mathrm{~s}$ & 23.7 \\
\hline Dashen & $\mathrm{B}$ & 30 & $s$ & 30 & $\mathrm{~ms}$ & 50 & $\mathrm{~s}$ & 36.7 & 20 & $\mathrm{~s}$ & 10 & $\mathrm{~s}$ & 40 & $\mathrm{~s}$ & 23.3 \\
\hline Mitike & $\mathrm{B}$ & 50 & $s$ & 10 & $\mathrm{~ms}$ & 50 & $\mathrm{~s}$ & 36.7 & 15 & $\mathrm{~s}$ & 20 & $\mathrm{~s}$ & 60 & $\mathrm{~s}$ & 31.7 \\
\hline Galema & B & 40 & $\mathrm{~s}$ & 15 & $\mathrm{~s}$ & 30 & $\mathrm{~s}$ & 28.3 & 5 & $\mathrm{~ms}$ & 20 & $\mathrm{~s}$ & 50 & $\mathrm{~s}$ & 25 \\
\hline Kubsa & $\mathrm{B}$ & 40 & $s$ & 30 & sms & 60 & $\mathrm{~s}$ & 43.3 & 10 & $\mathrm{~ms}$ & 30 & mss & 70 & $\mathrm{~s}$ & \begin{tabular}{|l|}
36.7 \\
\end{tabular} \\
\hline Abola & $\mathrm{B}$ & 10 & $\mathrm{~ms}$ & 1 & tmr & 60 & $\mathrm{~s}$ & 23.7 & 10 & sms & 30 & $\mathrm{~ms}$ & 70 & $\mathrm{~s}$ & \begin{tabular}{|l|}
36.7 \\
\end{tabular} \\
\hline Megal & $\mathrm{B}$ & 60 & $\mathrm{~S}$ & 10 & $\mathrm{~s}$ & 80 & $\mathrm{~s}$ & 50 & 30 & $\mathrm{~s}$ & 80 & $\mathrm{~S}$ & 60 & $\mathrm{~s}$ & 56.7 \\
\hline Tusie & $\mathrm{B}$ & 5 & s-ms & 1 & $\operatorname{tr}$ & 5 & $\mathrm{mr}$ & 3.7 & 5 & $\mathrm{~ms}$ & 20 & $\mathrm{~s}$ & 20 & $s$ & 15 \\
\hline Katar & $\mathrm{B}$ & 40 & $\mathrm{~s}$ & 5 & sms & 50 & $\mathrm{~S}$ & 31.7 & 5 & mss & 40 & $\mathrm{~S}$ & 60 & $\mathrm{~s}$ & 35 \\
\hline Shina & $\mathrm{B}$ & 60 & $\mathrm{~s}$ & 15 & $\mathrm{~s}$ & 80 & $\mathrm{~S}$ & 51.7 & 30 & $\mathrm{~s}$ & 20 & $\mathrm{~s}$ & 80 & $\mathrm{~s}$ & 43.3 \\
\hline Tura & $\mathrm{B}$ & 40 & $\mathrm{~s}$ & 1 & tms & 40 & $\mathrm{~S}$ & 27 & 5 & $\mathrm{~ms}$ & 20 & $\mathrm{~s}$ & 30 & $\mathrm{~s}$ & 18.3 \\
\hline Hawi & $\mathrm{B}$ & 20 & $s$ & 1 & $\operatorname{tmr}$ & 80 & $\mathrm{~S}$ & 33.7 & 10 & $s$ & 10 & $s$ & 70 & $s$ & 30 \\
\hline Madda Walabu & $\mathrm{B}$ & 1 & tr-ms & 10 & msmr & 60 & $\mathrm{~S}$ & 23.7 & 1 & tms & 20 & $S$ & 50 & $\mathrm{~s}$ & 23.7 \\
\hline Simba & B & 5 & sms & 10 & sms & 50 & $S$ & 21.7 & 5 & $\mathrm{~ms}$ & 20 & $\mathrm{~s}$ & 40 & $\mathrm{~s}$ & 21.7 \\
\hline Sofumar & $\mathrm{B}$ & 20 & sms & 1 & tms & 60 & $S$ & 27 & 1 & tms & 15 & $\mathrm{~s}$ & 50 & $\mathrm{~s}$ & 22 \\
\hline Wetera & B & 40 & $\mathrm{~s}$ & 5 & $\mathrm{~s}$ & 50 & $S$ & 31.7 & 5 & & 5 & $\mathrm{~ms}$ & 30 & $\mathrm{~s}$ & 13.3 \\
\hline Dodota & $\mathrm{B}$ & 50 & $\mathrm{~s}$ & 15 & $\mathrm{~s}$ & 20 & $\mathrm{~S}$ & 28.3 & 10 & & 0 & & 60 & $\mathrm{~s}$ & 23.3 \\
\hline Dur & $\mathrm{B}$ & 0 & 0 & . & 0 & 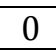 & - & 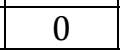 & 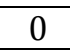 & 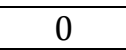 & 0 & & 0 & $\mathrm{~s}$ & 0 \\
\hline KBG-01 & $\mathrm{B}$ & 1 & & 1 & 0 & 60 & $\mathrm{~S}$ & 20.7 & 1 & $\mathrm{tm}$ & 40 & S & 60 & $s$ & 33.7 \\
\hline Sirbo & $\mathrm{B}$ & 30 & $\mathrm{~s}$ & 10 & $\mathrm{~s}$ & 50 & - & 30 & 1 & tms & 30 & $\mathrm{~S}$ & 20 & $\mathrm{~s}$ & 17 \\
\hline Tossa & $\mathrm{B}$ & 30 & $\mathrm{~S}$ & 1 & tms & 80 & $\mathrm{~S}$ & 37 & 5 & $\mathrm{~ms}$ & 5 & $\mathrm{~s}$ & 60 & $\mathrm{~s}$ & 23.3 \\
\hline Meraro & $\mathrm{B}$ & 10 & $s$ & 1 & tmr & 30 & $S$ & 13.7 & 1 & tms & 1 & tmr & 30 & $\mathrm{~s}$ & 10.7 \\
\hline Senkegna & $\mathrm{B}$ & 20 & $\mathrm{~s}$ & 1 & $\mathrm{tmr}$ & 10 & $\mathrm{~S}$ & 10.3 & 1 & tms & 5 & $\mathrm{~s}$ & 20 & $\mathrm{~s}$ & 8.7 \\
\hline Tay & $\mathrm{B}$ & 1 & ts & 0 & 0 & 5 & $\mathrm{~ms}$ & 2 & 5 & $\mathrm{~ms}$ & 40 & $\mathrm{~s}$ & 10 & $\mathrm{~s}$ & 18.3 \\
\hline Sulla & B & 20 & $\mathrm{~s}$ & 1 & tmr & 80 & $\mathrm{~S}$ & 33.7 & 30 & $\mathrm{~s}$ & 1 & ts & 80 & $\mathrm{~s}$ & 37 \\
\hline Alidoro & $\mathrm{B}$ & 1 & tms & 1 & $\operatorname{tr}$ & 60 & $S$ & 20.7 & 1 & Ts & 30 & $\mathrm{~s}$ & 5 & $\mathrm{~s}$ & 12 \\
\hline Millennium & $\mathrm{B}$ & 10 & $\mathrm{~S}$ & 0 & & 80 & $\mathrm{~S}$ & 30 & 1 & tms & 1 & tmr & 60 & $\mathrm{~s}$ & 20.7 \\
\hline Dinknesh & $\mathrm{B}$ & 5 & mss & 1 & $\operatorname{tr}$ & 1 & $\mathrm{~ms}$ & 2.3 & 1 & tms & 30 & $S$ & 5 & $\mathrm{~S}$ & 12 \\
\hline Menze & B & 5 & $\mathrm{~S}$ & 0 & & 90 & $\mathrm{~S}$ & 31.7 & 1 & ts & 20 & $\mathrm{~s}$ & 50 & $s$ & 23.7 \\
\hline Kulkulu & B & 20 & $\mathrm{~ms}$ & 5 & $\mathrm{~ms}$ & 80 & $S$ & 35 & 20 & $\mathrm{~s}$ & 30 & $\mathrm{~S}$ & 50 & $\mathrm{~s}$ & 33.3 \\
\hline Bolo & $\mathrm{B}$ & 1 & tms & 1 & $\operatorname{tmr}$ & 90 & $\mathrm{~S}$ & 30.7 & 1 & tms & 10 & $\mathrm{~ms}$ & 40 & $\mathrm{~s}$ & 17 \\
\hline Danda'a & B & 10 & $\mathrm{~S}$ & 5 & $\mathrm{mr}$ & 30 & $\mathrm{~S}$ & 15 & 5 & $\mathrm{mr}$ & 20 & $\mathrm{~s}$ & 20 & $\mathrm{~s}$ & 15 \\
\hline
\end{tabular}




\section{Open Access Journal of Agricultural Research}

\begin{tabular}{|c|c|c|c|c|c|c|c|c|c|c|c|c|c|c|c|}
\hline Kakaba & $\mathrm{B}$ & 30 & $\mathrm{~S}$ & 1 & $\operatorname{tr}$ & 80 & $\mathrm{~s}$ & 37 & 5 & mrms & 30 & $\mathrm{~s}$ & 40 & $\mathrm{~s}$ & 25 \\
\hline Hoggana & $\mathrm{B}$ & 5 & $\mathrm{~S}$ & 5 & $\mathrm{~ms}$ & 60 & $\mathrm{~S}$ & 23.3 & 5 & $\mathrm{~ms}$ & 10 & $\mathrm{~ms}$ & 50 & $\mathrm{~S}$ & 21.7 \\
\hline Shorima & $\mathrm{B}$ & 10 & $\mathrm{~s}$ & 1 & ts & 40 & $\mathrm{~s}$ & 17 & 5 & mss & 10 & $\mathrm{mss}$ & 50 & $\mathrm{~s}$ & 21.7 \\
\hline Huluka & B & 1 & tms & 5 & $\mathrm{~ms}$ & 40 & $\mathrm{~s}$ & 15.3 & 5 & $\mathrm{~ms}$ & 10 & $\mathrm{~s}$ & 30 & $\mathrm{~s}$ & 15 \\
\hline Gambo & $\mathrm{B}$ & 10 & $\mathrm{~ms}$ & 1 & $\operatorname{tr}$ & 40 & $\mathrm{~s}$ & 17 & 5 & $\mathrm{msmr}$ & 5 & sms & 40 & $\mathrm{~s}$ & 16.7 \\
\hline Galil & B & 80 & $\mathrm{~s}$ & 1 & $\operatorname{tr}$ & 60 & $\mathrm{~s}$ & 47 & 5 & $\mathrm{mr}$ & 1 & tms & 20 & $\mathrm{~s}$ & 8.67 \\
\hline Jafersson & $\mathrm{B}$ & 40 & $\mathrm{~s}$ & 1 & tms & 60 & $\mathrm{~s}$ & 33.7 & $\mathrm{n}$ & $\mathrm{n}$ & 1 & $\operatorname{tr}$ & 40 & $\mathrm{~s}$ & 20.5 \\
\hline Tsehay & $\mathrm{B}$ & 50 & $\mathrm{~s}$ & 5 & $\mathrm{msmr}$ & 70 & $\mathrm{~s}$ & 41.7 & 10 & $\mathrm{~s}$ & 0 & 0 & 5 & $\mathrm{~s}$ & 5 \\
\hline HAR 727 & $\mathrm{~B}$ & 5 & $\mathrm{~ms}$ & 1 & $\operatorname{tr}$ & 1 & $\mathrm{mr}$ & 2.3 & 1 & tmr & 20 & $\mathrm{~s}$ & 0 & & 7 \\
\hline HAR 723 & $\mathrm{~B}$ & 5 & $\mathrm{~ms}$ & 1 & $\operatorname{tr}$ & 80 & $\mathrm{~s}$ & 28.7 & 40 & $\mathrm{~s}$ & 1 & tms & 80 & $\mathrm{~s}$ & 40.3 \\
\hline HAR 934 & B & 10 & $\mathrm{~ms}$ & 10 & $\mathrm{mr}$ & 1 & $\mathrm{mr}$ & 7 & 1 & tms & 20 & $\mathrm{~s}$ & 0 & & 7 \\
\hline HAR 1018 & $\mathrm{~B}$ & 5 & $\mathrm{~ms}$ & 0 & & 5 & $\mathrm{mr}$ & 3.3 & 20 & sms & 5 & $\mathrm{~ms}$ & 40 & $\mathrm{~s}$ & 21.7 \\
\hline HAR 820 & $\mathrm{~B}$ & 10 & $\mathrm{~ms}$ & 1 & tms & 20 & $\mathrm{mr}$ & 10.3 & 10 & $\mathrm{~ms}$ & 5 & $\mathrm{~s}$ & 10 & $\mathrm{~ms}$ & 8.3 \\
\hline HAR 1407 & $\mathrm{~B}$ & 5 & $\mathrm{~ms}$ & 5 & $\mathrm{~ms}$ & 15 & $\mathrm{~ms}$ & 8.3 & 10 & $\mathrm{~ms}$ & 40 & $\mathrm{~s}$ & 10 & $\mathrm{~ms}$ & 20 \\
\hline HAR 1331 & $\mathrm{~B}$ & 10 & $\mathrm{~ms}$ & 1 & $\operatorname{tr}$ & 10 & $\mathrm{mr}$ & 7 & 0 & 0 & 30 & $\mathrm{~s}$ & & 0 & 15 \\
\hline HAR 719 & $\mathrm{~B}$ & 10 & $\mathrm{~ms}$ & 0 & 0 & 30 & $\mathrm{~S}$ & 13.3 & 0 & 0 & 10 & $\mathrm{~ms}$ & 50 & $\mathrm{~s}$ & 20 \\
\hline Hidassie & $\mathrm{B}$ & 15 & $\mathrm{~ms}$ & 1 & $\mathrm{tmr}$ & 50 & $\mathrm{~S}$ & 22 & 10 & msmr & 30 & $\mathrm{~S}$ & 40 & $\mathrm{~s}$ & 26.7 \\
\hline Ogolcho & $\mathrm{B}$ & 50 & $\mathrm{~S}$ & 5 & $\mathrm{mr}$ & 50 & $\mathrm{~S}$ & 35 & 10 & $\mathrm{~S}$ & 30 & $\mathrm{~S}$ & 20 & $\mathrm{~S}$ & 20 \\
\hline ETBW5800 & $\mathrm{B}$ & 20 & $\mathrm{~s}$ & 1 & tmr & 20 & $\mathrm{~S}$ & 13.7 & 1 & tmr & 30 & $\mathrm{~S}$ & 20 & $\mathrm{~S}$ & 17 \\
\hline ETBW5879 & $\mathrm{B}$ & 15 & $\mathrm{~s}$ & 1 & tms & 30 & $\mathrm{~s}$ & 15.3 & 10 & $\mathrm{~ms}$ & 40 & $\mathrm{~s}$ & 20 & $\mathrm{~s}$ & 23.3 \\
\hline ETBW5890 & $\mathrm{B}$ & 40 & $\mathrm{~s}$ & 1 & tmr & 60 & $\mathrm{~s}$ & 33.7 & 10 & $\mathrm{~ms}$ & 50 & $\mathrm{~s}$ & 10 & $\mathrm{~ms}$ & 23.3 \\
\hline ETBW6093 & $\mathrm{B}$ & 10 & $\mathrm{~ms}$ & 5 & $\mathrm{msmr}$ & 80 & $\mathrm{~S}$ & 31.7 & 50 & $\mathrm{~S}$ & 50 & $\mathrm{~S}$ & 50 & $\mathrm{~s}$ & 50 \\
\hline ETBW6094 & $\mathrm{B}$ & 10 & $\mathrm{~ms}$ & 5 & $\mathrm{~ms}$ & 50 & $\mathrm{~S}$ & 21.7 & 10 & $\mathrm{~ms}$ & 30 & $\mathrm{~S}$ & 80 & $S$ & 40 \\
\hline ETBW6098 & $\mathrm{B}$ & 10 & $\mathrm{~ms}$ & 10 & $\mathrm{~ms}$ & 80 & $\mathrm{~s}$ & 33.3 & 5 & $\mathrm{~ms}$ & 10 & $\mathrm{~s}$ & 60 & $S$ & 25 \\
\hline Kingbird & $\mathrm{B}$ & & - & 5 & $\mathrm{msmr}$ & 40 & $S$ & 22.5 & - & & 20 & $S$ & 20 & $\mathrm{mr}$ & 20 \\
\hline Mandoyu & $\mathrm{B}$ & - & - & 20 & sms & 60 & $\mathrm{~s}$ & 40 & - & - & 30 & $\mathrm{~s}$ & 20 & $\mathrm{mr}$ & 25 \\
\hline Sanate & $\mathrm{B}$ & - & 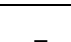 & 0 & & 15 & $\mathrm{~s}$ & 7.5 & - & 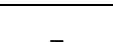 & 10 & $\mathrm{~s}$ & 1 & ts & 5.5 \\
\hline Gassay & $\mathrm{B}$ & & _ & 1 & $\operatorname{tmr}$ & 40 & $\mathrm{~s}$ & 20.5 & - & & 5 & $\mathrm{~ms}$ & 1 & $\operatorname{tmr}$ & 3 \\
\hline ETBW6647* & $\mathrm{B}$ & & - & 5 & $\mathrm{mr}$ & 80 & $\mathrm{~S}$ & 42.5 & - & & 30 & $\mathrm{~s}$ & 60 & $\mathrm{~s}$ & 45 \\
\hline ETBW6496* & $\mathrm{B}$ & - & - & 10 & $\mathrm{mss}$ & 30 & $S$ & 20 & - & - & 5 & $\mathrm{~ms}$ & 10 & $\mathrm{~s}$ & 7.5 \\
\hline ETBW6696* & $\mathrm{B}$ & - & _ & 5 & $\mathrm{mr}$ & 40 & $\mathrm{~s}$ & 22.5 & - & _ & 1 & $\mathrm{tmr}$ & 20 & $\mathrm{~s}$ & 10.5 \\
\hline ETBW7698* & $\mathrm{B}$ & _ & - & 1 & tms & 1 & $\mathrm{r}$ & 1 & - & _ & 10 & $\mathrm{~s}$ & 5 & $\mathrm{mr}$ & 7.5 \\
\hline ETBW6939* & $\mathrm{B}$ & _ & - & 5 & $\mathrm{~ms}$ & 20 & $\mathrm{~s}$ & 12.5 & - & - & 30 & $\mathrm{~s}$ & 40 & $\mathrm{~s}$ & 35 \\
\hline ETBW7255* & $\mathrm{B}$ & 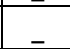 & & 0 & & 80 & $\mathrm{~S}$ & 40 & - & - & 60 & $\mathrm{~S}$ & 60 & $\mathrm{~S}$ & 60 \\
\hline ETBW6861* (Lemu) & $\mathrm{B}$ & & & 1 & tmr & 30 & $\mathrm{~S}$ & 15.5 & & & 30 & $S$ & 30 & $s$ & 30 \\
\hline \begin{tabular}{|l|} 
ICARDA ELITE 107 \\
\end{tabular} & $\mathrm{~B}$ & & & 1 & tmr & 15 & mss & 8 & & & 20 & $\mathrm{~S}$ & 20 & $\mathrm{~ms}$ & 20 \\
\hline AGUILAL/3/PYN & $\mathrm{B}$ & & & 5 & $\mathrm{~s}$ & 30 & $\mathrm{~s}$ & 17.5 & & & 20 & $\mathrm{~s}$ & 40 & $\mathrm{~s}$ & 30 \\
\hline ETBW6130 (Wane) & $\mathrm{B}$ & & & & & 10 & $\mathrm{mr}$ & 10 & & & & & 5 & $\mathrm{~s}$ & 5 \\
\hline Morocco & $\mathrm{B}$ & 80 & $\mathrm{~S}$ & 60 & $S$ & 80 & $\mathrm{~s}$ & & 60 & $\mathrm{~S}$ & 30 & $\mathrm{~S}$ & 80 & $S$ & \\
\hline PBW343 & $\mathrm{B}$ & 10 & $S$ & 1 & tms & 60 & $S$ & & 40 & $\mathrm{~S}$ & 40 & $S$ & 40 & $S$ & \\
\hline Digalu & $\mathrm{B}$ & 0 & $\mathrm{~s}$ & 1 & $\operatorname{tmr}$ & 70 & $\mathrm{~s}$ & & 1 & tms & 20 & $\mathrm{~s}$ & 60 & $\mathrm{~s}$ & \\
\hline
\end{tabular}

N.B: WT: Wheat Type, B-Bread, S-severity, R-reaction, ASV\% Average severity, Yr- yellow rust and (-) missing.

Table 1: The severity and reaction of commercial bread wheat and candidate varieties to yellow rust in 2014-2016 at Arsi Robe and Meraro.

\section{Reaction of Commercial Varieties of Durum Wheat to Yellow Rust}

Of the 21 durum wheat varieties included in the trap nursery, ten (Dure, Hitossa, Werer, Denbi, Mettaya, Ejersaa, Flakit, Toltu, Obssa, Tate and Bakalcha) showed below $10 \%$ average yellow rust severity with moderately resistant (MR) in terms of its reaction to yellow rust (Table 2). Whereas, Bobicho, Arendeto, Malefia and local red durum wheat verities exhibited a severity level range between $10-40 \%$. In general, yellow rust recorded in all cropping season in both crop (durum and bread wheat) and locations this indicated that in all season the yellow rust occurred at epidemic level and good opportunity to 


\section{Open Access Journal of Agricultural Research}

see the disease states of our commercial durum verities. Even though the yellow rust occur at higher levels most of our commercial durum varities shows resistant reaction for the given yellow rust isolates for the last three cropping season (Table2). Therefore, major of commercial durum varieties show resistance for yellow rust isolates than bread wheat.

\begin{tabular}{|c|c|c|c|c|c|c|c|c|c|c|c|c|c|c|c|}
\hline \multirow{4}{*}{ Variety/line } & \multirow{4}{*}{ WT } & \multirow{2}{*}{\multicolumn{6}{|c|}{$\begin{array}{c}\begin{array}{c}\text { Severity and Reaction of } \\
\text { Commercial Variety and Lines for } \\
\text { YR }\end{array} \\
\text { ARSI ROBE }\end{array}$}} & \multirow{4}{*}{ wASV\% } & \multirow{2}{*}{\multicolumn{6}{|c|}{$\begin{array}{c}\text { Severity and Reaction of } \\
\text { Commercial Variety and Lines for } \\
\text { YR }\end{array}$}} & \multirow{4}{*}{ ASV\% } \\
\hline & & & & & & & & & & & & & & & \\
\hline & & \multicolumn{2}{|c|}{2014} & \multicolumn{2}{|c|}{2015} & \multicolumn{2}{|c|}{2016} & & \multicolumn{2}{|c|}{2014} & \multicolumn{2}{|c|}{2015} & \multicolumn{2}{|c|}{2016} & \\
\hline & & $S$ & $\mathbf{R}$ & $\mathbf{S}$ & $\mathbf{R}$ & $\mathbf{S}$ & $\mathbf{R}$ & & $\mathbf{S}$ & $\mathbf{R}$ & $S$ & $\mathbf{R}$ & $\mathbf{S}$ & $\mathbf{R}$ & \\
\hline Arendeto & $\mathrm{D}$ & 5 & $\mathrm{~ms}$ & 0 & 0 & 40 & $S$ & 15 & 20 & $\mathrm{~s}$ & 0 & 0 & 10 & $\mathrm{~s}$ & 10 \\
\hline Hitossa & $\mathrm{D}$ & 0 & 0 & 0 & 0 & 1 & $\mathrm{~ms}$ & 0.3 & 0 & 0 & 1 & $\mathrm{tmr}$ & 1 & $\mathrm{~s}$ & 0.67 \\
\hline Werer & $\mathrm{D}$ & 0 & 0 & 0 & 0 & 0 & 0 & 0 & 0 & 0 & 1 & tmr & 1 & $\mathrm{~s}$ & 0.7 \\
\hline Denbi & $\mathrm{D}$ & 0 & 0 & 0 & 0 & 10 & $\mathrm{mr}$ & 3.3 & 1 & $\mathrm{tmr}$ & 1 & $\mathrm{tmr}$ & 1 & $\mathrm{~s}$ & 1 \\
\hline Selam & $\mathrm{D}$ & 10 & sms & 0 & 0 & 10 & $\mathrm{mr}$ & 6.7 & 10 & $\mathrm{~ms}$ & 5 & $\mathrm{mr}$ & 20 & $\mathrm{~s}$ & 11.7 \\
\hline Megenagna & $\mathrm{D}$ & 1 & tms & 1 & $\operatorname{tr}$ & 5 & $\mathrm{mr}$ & 2.3 & 5 & $\mathrm{msmr}$ & $\mathrm{N}$ & $\mathrm{N}$ & 20 & $\mathrm{~s}$ & 12.5 \\
\hline Mettaya & $\mathrm{D}$ & 1 & $\operatorname{Tr}$ & 0 & 0 & 0 & 0 & 0.3 & 1 & tmr & 1 & tmr & 1 & $\mathrm{~s}$ & 1 \\
\hline Ejersaa & $\mathrm{D}$ & 0 & 0 & 0 & 0 & 1 & $\mathrm{mr}$ & 0.3 & 0 & 0 & 1 & tmr & 1 & $\mathrm{~s}$ & 0.7 \\
\hline Flakit & $\mathrm{D}$ & 0 & 0 & 0 & 0 & 10 & $\mathrm{mr}$ & 3.3 & 0 & 0 & 20 & $\mathrm{~s}$ & 10 & $\mathrm{~s}$ & 10 \\
\hline Malefia & $\mathrm{D}$ & 10 & sms & 5 & $\mathrm{~ms}$ & 30 & $S$ & 15 & 10 & $\mathrm{~ms}$ & 10 & $\mathrm{~s}$ & 40 & $s$ & 20 \\
\hline Mossobo & $\mathrm{D}$ & 1 & $\operatorname{tmr}$ & 10 & $\mathrm{~s}$ & 5 & $\mathrm{mr}$ & 5.3 & 5 & $\mathrm{~ms}$ & 1 & tmr & 30 & $s$ & 12 \\
\hline Toltu & $\mathrm{D}$ & 1 & $\mathrm{tmr}$ & 1 & $\operatorname{tr}$ & 1 & $\mathrm{mr}$ & 1 & 0 & 0 & 1 & tms & 10 & $\mathrm{~s}$ & 3.7 \\
\hline Obssa & D & 0 & 0 & 0 & 0 & 0 & 0 & 0 & 0 & 0 & 5 & $\mathrm{~ms}$ & 5 & $\mathrm{~s}$ & 3.3 \\
\hline Lellisso & $\mathrm{D}$ & 5 & $\mathrm{~ms}$ & 1 & $\operatorname{tr}$ & 0 & 0 & 2 & 20 & $\mathrm{mr}$ & 1 & tms & 60 & $\mathrm{~s}$ & 27 \\
\hline Tate & $\mathrm{D}$ & 0 & 0 & 5 & $\mathrm{mr}$ & 5 & $\mathrm{mr}$ & 3.3 & 1 & tms & 1 & tms & 20 & $\mathrm{~s}$ & 7.3 \\
\hline Bakalcha & $\mathrm{D}$ & 0 & 0 & 0 & 0 & 10 & $\mathrm{mr}$ & 3.3 & 1 & $\mathrm{tmr}$ & 10 & $\mathrm{~s}$ & 10 & $\mathrm{~s}$ & 7 \\
\hline Oda & $\mathrm{D}$ & 1 & $\mathrm{tmr}$ & 5 & $\mathrm{mr}$ & 10 & $\mathrm{mr}$ & 5.3 & 10 & $\mathrm{~s}$ & 1 & tmr & 30 & $\mathrm{~s}$ & 13.7 \\
\hline Kokate & $\mathrm{D}$ & 1 & $\mathrm{tmr}$ & 1 & $\mathrm{tmr}$ & 5 & $\mathrm{mr}$ & 2.3 & 15 & $\mathrm{~s}$ & 1 & tms & 30 & $s$ & 15.3 \\
\hline Illani & D & 1 & $\mathrm{tmr}$ & 0 & & 5 & $\mathrm{mr}$ & 2 & 10 & $\mathrm{mr}$ & 40 & $\mathrm{~s}$ & 30 & $\mathrm{~s}$ & 26.7 \\
\hline Local Red & $\mathrm{D}$ & 60 & $s$ & 1 & ts & 0 & & 20.3 & 50 & $\mathrm{~s}$ & 1 & tmr & 60 & $\mathrm{~s}$ & 37 \\
\hline Bobicho & $\mathrm{B}$ & 40 & $\mathrm{~s}$ & 5 & $\mathrm{~ms}$ & 80 & $S$ & 41.7 & 10 & sms & 40 & $\mathrm{~s}$ & 50 & $\mathrm{~s}$ & 33.3 \\
\hline
\end{tabular}

WT- Wheat Type, D-Durum, S-severity, R-reaction, ASV\% Average severity, Yr- yellow rust

Table 2: The severity and reaction of commercial durum varieties to yellow rust in 2014-2016 at Arsi robe and Meraro.

\section{Reaction of Yellow Rust Differentials to Yellow Rust of Wheat in 2014-2016 Seasons}

Based on average severity percentage over years at Meraro virulence was detected for yellow rust resistance genes Yr1, Yr5, Yr6, Yr7, Yr8, Yr9, Yr 10, Yr17, Yr18, Yr26, Yr27 and YrA. Effective genes identified during the three year season were Yr15, Lassik (-Yr5) and YrSP (Table 3). This result in line with Woubit found Yr5, Yr15 and Yr26 genes effective against 20 stripe rust races originating from Ethiopia [9,11].

At Arsi Robe virulence was detected for Yr1, Yr5, Yr6, Yr7, Yr8, Yr9, Yr 10, Yr17, Yr18, Yr26, Yr27, YrA were not effective genes of yellow rust within three year season. Whereas genes Yr15 Lassik (-Yr5), Lassik (-Yr5) and AVOCET $S$ were effective to the yellow rust populations over year in Arsi Robe area. The highest average percentage of yellow rust severity of greater than 70 was recorded only on the checks (Morocco and Kubsa) (Table $3)$.

Meraro and Arsi robe has the widest virulence spectrum for yellow rust of wheat and as a result most of the yellow rust differentials were not effective to the yellow rust populations prevailing at Meraro and Arsi Robe have comparable virulence spectrum in each season 


\section{Open Access Journal of Agricultural Research}

of yellow rust and almost equal number of effective and ineffective genes were identified during the three year season in both locations. In general, compared to the other cropping seasons, the virulence spectrum of yellow rust has increased in 2015and 2016 years in all locations and the number of effective genes identified is very low in all locations. The virulence spectrum of yellow rust at
Meraro and Arsi-robe has always been wide because of the favorable environmental condition that prevailed there and the cereal monoculture (in terms of growing susceptible cultivars such as Kubsa and Digalu) that is widely practiced in Arsi zones which are among the major wheat-producing zones of the country.

\begin{tabular}{|c|c|c|c|c|c|c|c|c|c|}
\hline \multirow{3}{*}{ Line/ Verities } & \multirow{3}{*}{ Yr gene } & \multirow{2}{*}{\multicolumn{4}{|c|}{$\begin{array}{c}\text { Severity and Reaction of YR } \\
\text { MERARO }\end{array}$}} & \multirow{2}{*}{\multicolumn{4}{|c|}{$\begin{array}{c}\text { Severity and Reaction YR } \\
\text { ARSI ROBE }\end{array}$}} \\
\hline & & & & & & & & & \\
\hline & & 2014 & 2015 & 2016 & ASV\% & 2014 & 2015 & 2016 & ASV\% \\
\hline YR1/6* Avocet S & YR1 & T-ms & $70 \mathrm{~s}$ & $40 \mathrm{~s}$ & 36.97 & $50 \mathrm{~s}$ & ts & $80 \mathrm{~S}$ & 43.67 \\
\hline YR5/6* AOC CX86.6.1.20 & YR5 & T-ms & $60 \mathrm{~s}$ & $40 \mathrm{~s}$ & 33.68 & $30 \mathrm{~s}$ & $10 \mathrm{mss}$ & $50 \mathrm{~S}$ & 29.33 \\
\hline YR6/6* AOC CX94.2.2.25 & YR6 & $30 \mathrm{~s}$ & $80 \mathrm{~s}$ & $60 \mathrm{~s}$ & 56.67 & $70 \mathrm{~s}$ & $20 \mathrm{~s}$ & $80 \mathrm{~S}$ & 56.67 \\
\hline YR7/6* Avocet S & YR7 & $50 \mathrm{~s}$ & - & $60 \mathrm{~s}$ & 55 & $60 s$ & $30 \mathrm{~s}$ & $80 \mathrm{~S}$ & 56.67 \\
\hline YR8/6* Avocet S & YR8 & T-ms & $80 \mathrm{~s}$ & $20 \mathrm{~ms}$ & 32.27 & $10 \mathrm{~ms}$ & $10 \mathrm{~s}$ & $100 \mathrm{~S}$ & 39.33 \\
\hline YR9/6* Avocet S & YR9 & $40 s$ & $80 \mathrm{~s}$ & $50 \mathrm{~s}$ & 56.67 & $80 \mathrm{~s}$ & $25 s$ & $100 \mathrm{~S}$ & 68.33 \\
\hline YR10/6* Avocet S & YR10 & $20 \mathrm{~s}$ & $5 \mathrm{mr}$ & $60 \mathrm{~s}$ & 27.33 & $50 \mathrm{~s}$ & 0 & $20 \mathrm{~S}$ & 23.33 \\
\hline YR15/6* Avocet S & YR15 & 0 & 0 & Ts & 0.33 & ts & 0 & 0 & 0.33 \\
\hline YR17/3* AOC CX94.8.1.25 & YR17 & $15 \mathrm{~s}$ & $60 \mathrm{~s}$ & $20 \mathrm{~s}$ & 31.67 & $80 \mathrm{~s}$ & $5 \mathrm{mr}$ & $80 \mathrm{~S}$ & 54 \\
\hline YR18/3* AOC CX94.10.1.7 & YR18 & $30 \mathrm{~s}$ & $60 \mathrm{~s}$ & $80 \mathrm{~s}$ & 56.66 & $60 \mathrm{~s}$ & $15 \mathrm{~s}$ & $80 S$ & 51.67 \\
\hline YR26/3* AOC CX96.17.1. & YR26 & $50 \mathrm{~s}$ & $50 \mathrm{~s}$ & $80 \mathrm{~s}$ & 60 & $60 \mathrm{~s}$ & Ts & $70 \mathrm{~S}$ & 43.67 \\
\hline YRSP/6* AOC CX94.14.1.15 & YRSP & - & - & $10 \mathrm{~s}$ & 10 & $20 \mathrm{~s}$ & 0 & $30 \mathrm{~S}$ & 16.67 \\
\hline YR27/3* AOC CX94.19.1.1 & YR27 & $20 \mathrm{~ms}$ & $80 \mathrm{~s}$ & $60 \mathrm{~s}$ & 52 & $50 \mathrm{~s}$ & $\mathrm{~N}$ & $80 S$ & 65 \\
\hline AVOCET R & YRA & $60 s$ & $60 \mathrm{~s}$ & $80 \mathrm{~s}$ & 66.67 & $80 \mathrm{~s}$ & $40 \mathrm{~s}$ & $90 \mathrm{~S}$ & 70 \\
\hline AVOCET S & _- & _- & $20 \mathrm{~ms}$ & $40 \mathrm{~s}$ & 28 & _- & $10 \mathrm{mr}$ & $\mathrm{TR}$ & 2.5 \\
\hline Lassik(-Yr5) & (-YR5) & - & ts & 0 & 1 & _- & 0 & 0 & 0 \\
\hline Lassik(+Yr5) & (+YR5) & $\mathrm{N}$ & $10 \mathrm{~s}$ & $20 \mathrm{~s}$ & 15 & - & - & $10 \mathrm{~S}$ & 10 \\
\hline Morocco & & $60 s$ & $80 \mathrm{~s}$ & $80 \mathrm{~s}$ & 73.33 & $80 \mathrm{~s}$ & Tmr & $80 \mathrm{~S}$ & 53.47 \\
\hline Kubsa & & $60 \mathrm{~s}$ & $80 \mathrm{~s}$ & $80 \mathrm{~s}$ & 73.33 & -- & $15 \mathrm{~ms}$ & $60 \mathrm{~S}$ & 33 \\
\hline
\end{tabular}

ASV\% Average severity, YR- yellow rust and (-) missing

Table 3: Reaction of yellow rust differentials to yellow rust of wheat at Arsi Robe and Meraro 2014-2016.

\section{Conclusion}

The Bread wheat varieties such as HAR727, HAR934, Bonny, Sanate, ETBW7698, ETBW6130 (Wane), Enkoye, ET13A2, Meraro, Danda'a, Huluka, Gambo, and HAR719 was shows resistant to moderately resistant to the yellow rust within three consecutive season in both location under field condition. Majority of the durum varieties are resistant or moderately resistant to yellow rust like Dure, Hitossa, Werer, Denbi, Malefie, Mettaya, Ejersa, Flakit, Toltu, Obssa, Tate and Bakalcha. The above mentioned bread and durum wheat commercial verities advisable to be used by farmer to decreases the yield loss due to yellow rust for the coming cropping season. In addition, those beard and durum wheat varieties they can serve as important sources of resistance to the stripe rust races prevailing in Ethiopia and they should be included in the crossing blocks of the national wheat breeding research project.

From the yellow rust differentials line the Yr15 and Lassik (-Yr5) are effective genes at both locations within three consecutive season under field condition. These resistant Yr genes could be probably incorporated in 


\section{Open Access Journal of Agricultural Research}

wheat breeding programs either single or in combination with other genes through gene pyramiding providing the additive effects of several genes to offer the cultivar a wider base yellow rust resistance in particular and the rusts as a whole.

\section{Recommendation}

From commercial bread wheat cultivars such as Sanate, Wane, Enkoye, ET13A2, Meraro, Danda'a, Huluka, Gambo are resistant varieties for yellow rust in addition, the durum wheat varieties like Dure, Hitossa, Werer, Denbi, Malefie, Mettaya, Ejersa, Flakit was recommend to use by farmer. From differentials line such as Yr15 and Lassik (Yr5) are effective genes for yellow rust.

\section{Acknowledgement}

Ethiopian Institute of Agricultural Research (EIAR), Kulumasa Agricultural Research Center (KARC) is acknowledged for financial support in undertaking the research work.

\section{References}

1. Negassa M (1986) Estimates of phenotypic diversity and breeding potential of Ethiopian wheats. Hereditas 104(1): 41-48.

2. Hundie B, Kumbi S, Hailu D (2002) Seasonal variations in the occurrence of wheat yellow rust in Bale highlands. Pest Management Journal of Ethiopia 6: 65-72.

3. Hailu D, Fininsa C (2007) Epidemics of yellow rust (Puccinia striiformis) on common wheat (Triticumaestivum) in the highlands of Bale, southern Ethiopia. Crop Protection 26: 1209-1218.

4. Hailu D, Fininsa C (2009) Relationship between stripe rust (Puccinia striiformis) and common wheat
(Triticumaestivum) yield loss in the highlands of Bale, southeastern Ethiopia. Archives of Phytopathology and Plant Protection 42(6): 508-523.

5. Ayele BH (2002) Breeding bread wheat with multiple disease resistance and high yield for the Ethiopian highlands: broadening the genetic basis of yellow rust and tan spot resistance. Ph D Thesis, Georg-August University pp: 132.

6. Ayele B, Stubbs RW (1995) Valuable sources of resistance of wheat to the East African yellow rust isolates. In: Danial DL (eds.) Breeding for disease resistance with emphasis on durability. Proceedings of a regional workshop for Eastern, Central, and Southern Africa pp: 206-214.

7. Roelfs AP, Singh RP, Saari EE (1992) Rust diseases of wheat: Concepts and methods of disease management. CIMMYT pp: 81.

8. Peterson RF, Campbell AB, Hannah AE (1948) A diagrammatic scale for estimating rust intensity of leaves and stems of cereals. Canadian Journal of Research 26c(5): 496-500.

9. Woubit D (2008) Analysis of pathogen virulence and cultivar resistance to stripe rust, Puccinia striiformis f. sp. tritici in Ethiopia. PhD Thesis pp: 100.

10. Ayele B, Wondimu B (1992) The importance of yellow rust in the major bread wheat producing regions of Ethiopia in 1988-90. In: The 7th Regional Wheat Workshop for Eastern, Central and Southern Africa pp: 196-202.

11. Worku D (2014) Epidemics of Pucciniastriiformis F. Sp. Tritici in Arsi and West Arsi Zones of Ethiopia in 2010 and Identification of Effective Resistance Genes. Journal of Natural Sciences Research 4(7). 